\title{
On Approximation-Solvability of Nonlinear Equations in Reflexive Banach Spaces
}

\author{
R. VERMA and L. DEBnAth
}

We extend the Zarantonello numerical range to the case of reflexive Banach space operators, and study the approximation-solvability of nonlinear equations relating to the results of Zeidler (1990).

Key words: Approximation scheme, approximation-solvability, A-proper mappings

AMS subject classification: $65 \mathrm{~J} 15$

\section{Introduction}

We consider an inner approximation scheme $\pi_{0}=\left\{\ddot{X}_{n}, E_{n}, R_{n}, Y_{n}, Q_{n}\right\}$ represented by

$\begin{array}{ccc}X & \stackrel{A}{\rightarrow} & Y \\ R_{n} \downarrow \uparrow E_{n} & & \downarrow Q_{n} \\ X_{n} & \stackrel{A_{n}}{\rightarrow} & Y_{n}\end{array}$

\section{Diagram 1.1}

Here $X$ and $Y$ are two normed linear spaces, and $\left\{X_{n}\right\}$ and $\left\{Y_{n}\right\}$ are two sequences of finitedimensional normed linear spaces. Let $\left\{E_{n}\right\},\left\{R_{n}\right\}$ and $\left\{Q_{n}\right\}$ be three sequences of connection operators. All operators $A_{n}=Q_{n} A E_{n}$ are assumed to be nonlinear and continuous, where $A: X \rightarrow Y$ is nonlinear corresponding to the equation

$$
A x=b, x \in X, b \in Y \text {. }
$$

As far as the solvability of the equation (1.1) is concerned, we consider not just the usual solvability - the existence of a solution of equation (1.1) is somehow established, but an approximation-solvability - a solution of equation (1.1) is obtained as a limit of solutions of the simpler finite-dimensional problems

$$
A_{n} x_{n}=Q_{n} b \text { for } x_{n} \in X_{n}, Q_{n} b \in Y_{n} .
$$

Now the problem is: For what type of a linear or nonlinear mapping $A$, is it possible to construct a solution of equation (1.1) as a strong limit of solutions $x_{n}$ of equations (1.2)?

R. Verma: University of Central Florida, Dep. Math. , Orlando, FL 32816, USA

L. Debnath: University of Central Florida, Dep. Math. , Orlando, FL 32816, USA 
Browder and Petryshyn [1] developed $A$-proper mappings to solve this problem. They studied $A$-proper mappings in a series of publications, and that turned out closely connected with the approximation-solvability of equation (1.1). The theory of $A$-proper mappings does extend and unify results concerning the Galerkin type methods for linear and nonlinear equations in the theory of strongly monotone and accretive operators, operators of the type $(S), P_{\mathrm{v}}$-compact, ball condensing and other mappings. For details, we refer to [1-6] and [8]. The concept of an $A$-proper mapping extends also to the stability of the projectional method in the sense of Mikhlin, and relates to the solvability of elliptic partial differential equations. We extend the Zarantonello numerical range [7] to the case of reflexive Banach space operators, and establish the approximation-solvability of the related nonlinear equations. Some interesting special cases are also considered in a Hilbert space setting.

Before proceeding to the main results, we need to recall some definitions for the sake of the completeness.

The symbol $\mathbf{K}$ shall denote either the real or the complex field. In what follows, the symbol $" \rightarrow$ " shall denote strong convergence, and the symbol " $\rightarrow$ " shall denote weak convergence.

Definition 1.1. An approximation scheme $\pi_{0}=\left\{X_{n}, E_{n}, R_{n}, Y_{n}, Q_{n}\right\}$ is said to be compatible if $\lim _{n \rightarrow \infty}\left\|E_{n} R_{n} x-x\right\|_{X}=0 \forall x \in X$

consistent if $\lim _{n \rightarrow \infty}\left\|Q_{n} A x-A_{n} R_{n} x\right\|_{Y_{n}}=0 \forall x \in X$

stable if, for $d>0,\left\|A_{n} u-A_{n} v\right\|_{Y_{n}} \geq d|\|u\|-\|v\||_{X_{n}} \forall u, v \in X_{n}$

admissible inner, if it is compatible and the following conditions (C1) - (C3) hold:

(C1) $X$ and $Y$ are infinite-dimensional normed spaces over $\mathbf{K}$

(C2) $X_{n}$ and $Y_{n}$ are normed spaces over $\mathbf{K}$ with $\operatorname{dim} X_{n}=\operatorname{dim} Y_{n}<\infty$

(C3) $E_{n}$ and $Q_{n}$ are linear and continuous operators with $\sup \left\|E_{n}\right\|, \sup \left\|Q_{n}\right\|<\infty$.

Note that the definition of the stability here, a special case of the usual stability condition, is relevant to the present investigation.

Definition 1.2 (Solvability). The equation (1.1) is said to be solvable if it has a solution; and it is said to be uniquely approximation-solvable, if, for each $b \in Y$, the following conditions are satisfied:

(C4) The equation $A x=b, x \in X$, has a unique solution.

(C5) The approximate equations $A_{n} x_{n}=Q_{n} b, x_{n} \in X_{n}, n \geq n_{0}$, have unique solutions $x_{n}$. 
(C6) The solution sequence $\left\{x_{n}\right\}$ converges to the solution $x$ of the equation $A x=b$ in the sense that $\lim _{n \rightarrow \infty}\left\|E_{n} x_{n}-x\right\|_{x}=0$.

Definition 1.3 (A-Properness). An operator $A: \dot{X} \rightarrow Y$ is called A-proper with respect to the approximation scheme $\pi_{0}=\left\{X_{n}, E_{n}, R_{n}, Y_{n}, Q_{n}\right\}$ if the following holds:

If $\left\{x_{n}: x_{n} \in X_{n}\right\}$ is any subsequence such that $\left\|A_{n} x_{n}-Q_{n} b\right\|_{Y_{n}} \rightarrow 0$ for some $b \in Y$, and sup $\left\|x_{n}\right\|_{X_{n}}<\infty$, then there exists an infinite subsequence $\left\{x_{n_{k}}\right\}$ and an element $x \in X$ such that $E_{n_{k}} x_{n_{k}} \rightarrow x$ in $X($ as $k \rightarrow \infty)$ and $A x=b$.

Definition 1.4 (Duality mapping). We recall that a continuous function $\mu: \mathbf{R}^{+}=\{t: t \geq 0\} \rightarrow \mathbf{R}^{+}$is called a gauge function if $\mu(0)=0$, and $\mu$ is strictly increasing. Let $X$ be a real reflexive Banach space and $X^{*}$ its dual. We denote by $[\cdot, \cdot]$ the duality pairing between the elements of $X^{*}$ and $X$. A mapping $J: X \rightarrow X^{*}$ is said to be a duality mapping between $X$ and $X^{*}$ with respect to gauge function $\mu$ if .

(C7) $[J x, x]=\mu(\|x\|)\|x\|$, and $\|J x\|=\mu(\|x\|)$ for $x \in X$.

We note that if $\mu(t)=t, J$ is called a normalized duality mapping. If $X^{*}$ is strictly convex, then $J$ is uniquely determined by $\mu$, and if $X$ is also reflexive, then $J$ is a single-valued demicontinuous mapping of $X$ onto $X^{*}$, which is bounded and positively homogeneous; furthermore, $J$ is monotone and satisfies the property

(C8) $[J x-J y, x-y]=[J x, x-y]-[J y, x-y]$

$$
\geq|\mu(\|x\|)-\mu(\|y\|)|\|x-y\| \forall x, y \in X .
$$

If $J$ is a normalized duality, (C8) reduces to

(C9) $[J x-J y, x-y] \geq|\|x\|-\|y\||\|x-y\| \forall x, y \in X$.

If $X$ is strictly convex, then the operator $J: X \rightarrow X^{*}$ is strictly monotone and bijective. The inverse operator $J^{-1}: X^{*} \rightarrow X$ equals the duality mapping of the dual space $X^{*}$ provided that $X$ is reflexive. For more details on the duality mappings, see [8].

Let $X$ be a normed space, $x \in X$ an element and $\left\{x_{n}\right\},\left\{y_{n}\right\} \subset X$ sequences with $\|x\|=1$ and $\left\|x_{n}\right\|=\left\|y_{n}\right\|=1$ for all $n$. Then $X$ is said to be

uniformly convex if $x_{n}+y_{n} \rightarrow 2$ implies $x_{n}-y_{n} \rightarrow 0$

locally uniformly convex if $x_{n}+x \rightarrow 2$ implies $x_{n} \rightarrow x$.

In particular, each locally uniformly convex space is a 
Kadec space, that is $\left\|x_{n}\right\| \rightarrow\|x\|, x_{n} \stackrel{w}{\rightarrow} x$ implies $x_{n} \rightarrow x$.

Note that each Banach space, which is locally uniformly convex, is strictly convex.

Definition 1.5 (Numerical range). Let $X$ be a reflexive Banach space and $X^{*}$ its dual. The numerical range of an operator $A: X \rightarrow X^{*}$, denoted by $n[\dot{A}]$, is defined to be the set

$$
n[A]=\left\{\frac{[A x, x]+[A y-A z, y-z]}{[J x, x]+[J y-J z, y-z]}: x, y, z \in X, y \neq z\right\} .
$$

Here $J: X \rightarrow X^{*}$ is a strictly monotone duality. Clearly, $n[A]$ is a subset of $\mathbf{K}$, and when $X$ is a Hilbert space, it reduces to the numerical range [5] defined by

$$
V[A]=\left\{\frac{\langle A x, x\rangle+\langle A y-A z, y-z\rangle}{\|x\|^{2}+\|y \dot{-} z\|^{2}}: x, y, z \in X, y \neq z\right\},
$$

where $\langle\cdot, \cdot\rangle$ is the standard inner product on $X$. The numerical range $n[A]$ reduces to the special cases of the Zarantonello numerical range [7] when $A(0)=0$. It coincides with the usual numerical range when $A$ is linear and $y-z=x$.

\section{Approximation Schemes in Banach Spaces}

Let $A: X \rightarrow X^{*}$ be an operator from a separable reflexive complex Banach space $X$ to its dual $X^{*}$. Consider the operator equation $A x=b(x \in X)$ with approximate equations

$$
E_{n}^{*} A E_{n} x_{n}=E_{n}^{*} b\left(x_{n} \in X_{n}, b \in X^{*}(n \in \mathrm{N})\right) \text {. }
$$

corresponding to an approximation scheme $\pi_{1}=\left\{X_{n}, E_{n}, R_{n}, X_{n}^{*}, E_{n}^{*}\right\}$ represented by Diagram 2.1 .

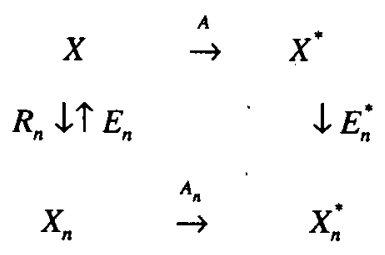

\section{Diạgram 2.1}

Here all $A_{n}=E_{n}^{*} A E_{n}$ are continuous. Let $\left\{X_{n}\right\}$ be a Galerkin scheme in $X$ with $X_{n}=\operatorname{span}\left\{e_{1 n}, \ldots, e_{n^{\prime} n}\right\}, n \in \mathbf{N}$ and $E_{n}: X_{n} \rightarrow X$. the embedding operator corresponding to $X_{n} \subseteq X$. Furthermore, for each $x \in X$, there exists at least one element $R_{n} x \in X_{n}$ such that $\left\|x-R_{n} x\right\|=\operatorname{dist}\left(x, X_{n}\right)$. For $n \in \mathbf{N}$, assume the approximate equations (2.1) are equivalent to Galerkin equations $\left[A x_{n}, e_{j n}\right]=\left[b, e_{j n}\right]\left(x_{n} \in X_{n} ; j=1,2, \ldots, n^{\prime}\right)$. We recall the following result, 
crucial to the inner approximation-solvability.

Lemma 2.1 [8, Proposition 34.9]. Let $A: X \rightarrow X^{*}$ be strongly monotone. If $\pi_{1}=\left\{X_{n}, E_{n}, R_{n}, X_{n}^{*}, E_{n}^{*}\right\}$ represents an admissible inner approximation scheme with consistency and stability, then the equation $A x=b, x \in X$, is uniquely approximation-solvable if and only if $A$ is $A$-proper. If, in addition, $C: X \rightarrow X^{*}$ is compact, then, for each $\lambda \neq 0, \lambda(A+C): X \rightarrow X^{*}$ is A-proper.

We first give results concerning elementary properties of the numerical range. Then we establish results on the solvability (inner approximation-solvability) of a class of nonlinear equations.

Theorem 2.1. Let $A, B: X \rightarrow X^{*}$ be mappings from a reflexive Banach space $X$ to its dual $X^{*}, J: X \rightarrow X^{*}$ a strictly monotone normalized duality, and $\lambda \in \mathbf{K}$. Then
(i) $n[\lambda A]=\lambda n[A]$
(ii) $n[A+B] \subset n[A]+n[B]$
(iii) $n[A-\lambda J]=n[A]-\{\lambda\}$

The proof follows from the definition.

Theorem 2.2. Let $X$ be a separable complex Banach space with dim $X^{-}<\infty$, and $X^{*}-i t s$ dual. If $X$ and $X^{*}$ are locally uniformly convex, $A: X \rightarrow X^{*}$ is continuous, $J: X \rightarrow X^{*}$ is a normalized duality, and $\lambda \in \mathbf{K}$ is at a positive distance $d$ from the numerical range of $A$, then, for each $b \in X^{*}$, the equation $A \dot{x}-\lambda J x=b(x \in X)$ has a unique solution.

Corollary 2.1 [5, Theorem 2.2]. Let $X$ be a separable Hilbert space over $\mathbf{K}, A: X \rightarrow X$ continuous, and $\lambda \in \mathbf{K}$ be at a positive distance from the numerical range $V[A]$ of $A$. Then, for each $b \in X$, the equation $A x-\lambda x=b, x \in X$, has a unique solution.

Proof of Theorem 2.2. Since $X$ is strictly convex (and hence $J: X \rightarrow X^{*}$ is strictly monotone), we have, for $x, y, z \in X$ with $y \neq z$,

$$
\begin{aligned}
|[(A-\lambda J) x, x]+[(A-\lambda J) y-(A-\lambda J) z, y-z]| \\
\quad=|[A x, x]+[A y-A z, y-z]-\lambda([J x, x]+[J y-J z, y-z])| \\
\quad=\left|\frac{[A x, x]+[A y-A z, y-z]}{[J x, x]+[J y-J z, y-z]}-\lambda\right||([J x, x]+[J y-J z, y-z])| \\
\quad \geq d \operatorname{Re}([J x, x]+[J y-J z, y-z]) \\
\quad \geq d\left(\|x\|^{2}+\|y-z\||\|y\|-\|z\||\right) .
\end{aligned}
$$

For $x=0$, this inequality reduces to the fundamental inequality 


$$
|[(A-\lambda J) y-(A-\lambda J) z, y-z]| \geq d\|y-z\||\|y\|-\|z\||
$$

for all $y, z \in X$. This implies that

$$
\|(A-\lambda J) y-(A-\lambda J) z\| \geq d\|\| y\|-\| z\|\|
$$

for all $y, z \in X$. Since $X$ is strictly convex, it follows immediately from here that $A-\lambda J$ is one-to-one. Let us take $c(r)=d r-\|(A-\lambda J)(0)\|$. Then, for $x \in X$, we obtain from (2.3) that

$$
\begin{aligned}
|[(A-\lambda J) y, y]| & \geq|[(A-\lambda J) y-(A-\lambda J)(0), y]|-|[(A-\lambda J)(0), y]| \\
& \geq d\|y\|^{2}-\|(A-\lambda J)(0)\|\|y\| \\
& =c(\|y\|)\|y\|,
\end{aligned}
$$

and so $\|(A-\lambda J) y\| \geq c(\|y\|)$ for $y \neq 0$. For each $M>0$, therefore, there exists $k(M)$ such that if $\|(A-\lambda J) y\| \leq M$, then $\|y\| \leq k(M)$. Thus, $(A-\lambda J)^{-1}$ carries bounded subsets of $R(A-\lambda J)$ into bounded subsets of $X$, and is continuous. By the Brouwer theorem on invariance of domain, $R(A-\lambda J)$ is open. To this end, it only remains to show that $R(A-\lambda J)$ is closed. To prove this, let $(A-\lambda J) x_{m} \rightarrow b$. Thus, $\left\{(A-\lambda J) x_{m}\right\}$ is a Cauchy sequence. Since $X$ is finitedimensional, there exists a subsequence, again denoted by $\left\{x_{n}\right\}$, such that, for some $x \in X$, $x_{m} \rightarrow x$. Hence, by the continuity of $A-\lambda J$, we obtain $(A-\lambda J) x=b$, and so $b \in R(A-\lambda J)$. Thus, the nonempty set $R(A-\lambda J)$ is both open and closed, and hence $R(A-\lambda J)=X^{*}$ and $A-\lambda J$ is bijective.

Theorem 2.3. Let $X$ be a separable reflexive complex Banach space with $\operatorname{dim} X=\infty$ and $X^{*}$ its dual. If $X$ and $X^{*}$ are locally uniformly convex, $A: X \rightarrow X^{*}$ is continuous, $J: X \rightarrow X^{*}$ is a normalized duality, and $\lambda \in \mathbf{K}$ is at a positive distance $d$ from the numerical range of $A$, then, for each $b \in X^{*}$, the equation $A x-\lambda J x=b, x \in X$, is uniquely approximation-solvable.

Proof. We prove the theorem by an application of Lemma 2.1. To do this, we first show that $\pi_{1}=\left\{X_{n}, E_{n}, R_{n}, X_{n}^{*}, E_{n}^{*}\right\}$ is an admissible inner approximation scheme. Since $\left\|E_{n}\right\|=1$, this implies that $\left\|E_{n}^{*}\right\|=1$ for all $n$ and since $\left\{X_{n}\right\}$ is a Galerkin scheme, we obtain $\operatorname{dist}\left(x, X_{n}\right) \rightarrow 0$, for all $x \in X$. Thus, $\left\|R_{n} x-x\right\| \rightarrow 0$, and the compatibility condition is satisfied.

Consistency: Since $A$ (and $A-\lambda J$ ) is continuous, the compatibility condition implies that $\left\|(A-\lambda J) E_{n} R_{n} x-(A-\lambda J) x\right\| \rightarrow 0$. Since sup $\left\|E_{n}^{*}\right\|<\infty$, we obtain (as $\left.n \rightarrow \infty\right)$

$$
\begin{aligned}
\left\|E_{n}^{*}(A-\lambda J) x-A_{n} R_{n} x\right\| & =\left\|E_{n}^{*}(A-\lambda J) x-E_{n}^{*}(A-\lambda J) E_{n} R_{n} x\right\| \\
& \leq\left\|E_{n}^{*}\right\|\left\|(A-\lambda J) x-(A-\lambda J) E_{n} R_{n} x\right\| \rightarrow 0 .
\end{aligned}
$$

Stability: By inequality (2.3), we obtain 


$$
\begin{aligned}
\left\|A_{n} x-A_{n} y\right\|\|x-y\| & \geq\left|\left[A_{n} x-A_{n} y, x-y\right]\right| \\
& =\left|\left[E_{n}^{*}(A-\lambda J) E_{n} x-E_{n}^{*}(A-\lambda J) E_{n} y, x-y\right]\right| \\
& =\left|\left[(A-\lambda J) E_{n} x-(A-\lambda J) E_{n} y, E_{n} x-E_{n} y\right]\right| \\
& =|[(A-\lambda J) x-(A-\lambda J) y, x-y]| \\
& \geq d|\|x\|-\|y\||\|x-y\|
\end{aligned}
$$

for all $x, y \in X_{n}$. This implies that $\left\|A_{n} x-A_{n} y\right\| \geq d|\|x\|-\|y\||$ for all $\dot{x}, y \in X_{n}$.

A-Properness: To show that $A-\lambda J$ is $A$-proper with respect to the approximation scheme $\pi_{1}=\left\{X_{n}, E_{n}, R_{n}, X_{n}^{*}, E_{n}^{*}\right\}$, let $\sup \left\|x_{n}\right\|<\infty$ for some $x_{n} \in X_{n}$ such that $\left\|A_{n} x_{n}-E_{n}^{*} b\right\|=$ $\left\|E_{n}^{*}(A-\lambda J) x_{n}-E_{n}^{*} b\right\| \rightarrow 0$. Since $X$ is reflexive and separable, there exists a subsequence, again denoted by $\left\{x_{n}\right\}$, such that, for some $x \in X, x_{n} \stackrel{w}{\rightarrow} x$ in $X$. Since $\left\|R_{n} x-x\right\| \rightarrow 0, x_{n} \rightarrow x$ implies that

$$
x_{n}-R_{n} x \stackrel{w}{\rightarrow} 0
$$

Also, we obtain (as $n \rightarrow \infty)$

$$
b=(A-\lambda J) R_{n} x \rightarrow b-(A-\lambda J) x .
$$

It would suffice to show that $x_{n} \rightarrow x$ and $(A-\lambda J) x=b$. From (2.5) and (2.6), it follows, for some $x_{n} \in X_{n}$ as above, that (as $n \rightarrow \infty$ )

$$
\begin{aligned}
d\left|\left\|x_{n}\right\|-\left\|R_{n} x\right\|\right| \| & x_{n}-R_{n} x \| \leq\left|\left[A_{n} x_{n}-A_{n} R_{n} x, x_{n}-R_{n} x\right]\right| \\
& =\left|\left[E_{n}^{*}(A-\lambda J) x_{n}-E_{n}^{*}(A-\lambda J) R_{n} x, x_{n}-R_{n} x\right]\right| \\
& =\left|\left[E_{n}^{*}(A-\lambda J) x_{n}-E_{n}^{*} b+E_{n}^{*} b-E_{n}^{*}(A-\lambda J) R_{n} x, x_{n}-R_{n} x\right]\right| \\
& =\left|\left[E_{n}^{*}(A-\lambda J) x_{n}-E_{n}^{*} b, x_{n}-R_{n} x\right]+\left[E_{n}^{*} b-E_{n}^{*}(A-\lambda J) R_{n} x, x_{n}-R_{n} x\right]\right| \\
& =\left|\left[E_{n}^{*}(A-\lambda J) x_{n}-E_{n}^{*} b, x_{n}-R_{n} x\right]+\left[b-(A-\lambda J) R_{n} x, E_{n} x_{n}-E_{n} R_{n} x\right]\right| \\
& =\left|\left[E_{n}^{*}(A-\lambda J) x_{n}-E_{n}^{*} b, x_{n}-R_{n} x\right]+\left[b-(A-\lambda J) R_{n} x, x_{n}-R_{n} x\right]\right| \rightarrow 0 .
\end{aligned}
$$

Since $\left\|R_{n} x-x\right\| \rightarrow 0$, it follows from $\left\|x_{n}\right\|-\left\|R_{n} x\right\| 1 \rightarrow 0$ that $\left\|x_{n}\right\| \rightarrow\|x\|$. Since $X$ is locally uniformly convex, $x_{n} \stackrel{w}{\rightarrow} x$ and $\left\|x_{n}\right\| \rightarrow\|x\|$ implies that $x_{n} \rightarrow x$. Hence, $(A-\lambda J) x=b$ by the continuity of $A$ (and hence $A-\lambda J$ ), and the theorem follows from Lemma 2.1 .

Corollary to Theorem 2.3 [5, Theorem 2.2]. Let $X$ be a separable complex Hilbert space with $\operatorname{dim} X=\infty$, and $A: X \rightarrow X$ be continuous. If $\lambda \in \mathbf{K}$ is at a positive distance from the numerical range [5] of $A$ (that is $d=\operatorname{dist}(\lambda, V[A])>0$ ), then, for each $b \in X$, the equation $A x-\lambda x=b, x \in X$, is uniquely approximation-solvable. 
Acknowledgements. This work was supported by a research grant of the second author from the University of Central Florida. The authors wish to express their appreciation to the referees and the redaction for helpful suggestions.

\section{REFERENCES}

[1] BROWDER, F.E. and W. PETRYSHYN: Approximation methods and the generalized topological degree for nonlinear mappings in Banach spaces. J. Funct. Anal. 3 (1969), $217-245$.

[2] DeIMLING, K.: Nonlinear Functional Analysis. New York: Springer-Verlag 1985.

[3] FITZPATRICK, P.M.: On the structure of the set of solutions of equations involving A-proper mappings. Trans. Amer. Math. Soc. 189 (1974), 107 - 131.

[4] PETRYSHYN, W.: On the approximation-solvability of equations involving A-proper and pseudo-A-proper mappings. Bull. Amer. Math. Soc. 81 (1975), 223 - 312.

[5] VERMA, R.: Role of numerical range in approximation-solvability of nonlinear functional equations. Appl. Math. Lett. 5 (1992), 25 - 27.

[6] VERMA, R.: Numerical range and related nonlinear equations. Czech. Math. J. (to appear).

[7] ZARANTONELLO, E.: The closure of the numerical range contains the spectrum. Pac. J. Math. 22 (1967), 575 - 595.

[8] ZeIDLER, E.: Nonlinear Functional Analysis, Part II B. New York: Springer - Verlag 1990. 